\section{Laboratorio internacional de paisajes culturales}

En septiembre de 2001 varios profesores e investigadores de la Universidad Politécnica de Cataluña y del Massachusetts Institute of Technology, fundaron un Laboratorio Internacional para la investigación e impulso de los paisajes culturales. Este Laboratorio vino avalado por diversos proyectos previos de colaboración en el campo de la investigación y el desarrollo y obtuvo el apoyo del Departamento de Universidades, Investigación y Sociedad de la Información de la Generalitat de Cataluña, de algunas instituciones (COAC, AADIPA, MNATEC), de profesionales y de estudiosos de los paisajes culturales. A su vez la Diputación de Barcelona nos confió diversos estudios de revaloración de los recursos patrimoniales a lo largo de los principales cursos fluviales de la provincia de Barcelona.

El Laboratorio pretende reclamar la atención sobre la proyectación urbanística basada en los recursos culturales. Se trata de una aproximación relativamente novedosa en Europa y los Estados Unidos, que no ha sido hasta la fecha objeto de la necesaria atención. Los miembros de ambas Universidades habían desarrollado durante los dos años previos un primer análisis de diversas experiencias en todo el mundo, que dió como fruto la publicación de un libro, donde por vez primera fueron descritos y analizados numerosos paisajes culturales y parques patrimoniales (Proyectando el Eje del Llobregat, Paisaje cultural y desarrollo regional. Barcelona, 2001). Este trabajo supuso al mismo tiempo un intento de deducir algunas lecciones de este nuevo enfoque del planeamiento y criterios para su correcta aplicación.

Las iniciativas basadas en los recursos culturales y el patrimonio en su sentido más amplio están proliferando en los últimos años. Bastantes de estos proyectos implican la adaptación de antiguas áreas industriales y agrícolas a los nuevos requerimientos y actividades propias del siglo XXI (nuevas tecnologías, educación, comunicaciones, ocio y turismo, información...).

En Europa se están desarrollando numerosos proyectos. Valgan como ejemplo los del Emscher Park en la región industrial del Ruhr; los de las áreas mineras de la Borgoña francesa y de la Toscana; o la recuperación de viejas colonias industriales inglesas o catalanas. Conviene asimismo mencionar los parques agrarios de Milán y Palermo en Italia, o del Llobregat en Cataluña.

En los Estados Unidos se han reconocido ya un centenar de proyectos de parques patrimoniales y paisajes culturales regionales o locales, impulsados o amparados por programas federales y estatales.

El Laboratorio Internacional trata de interpretar, alentar y difundir estas experiencias, con el objetivo de extender la atención al patrimonio cultural como factor fundamental en la construcción del territorio y de la

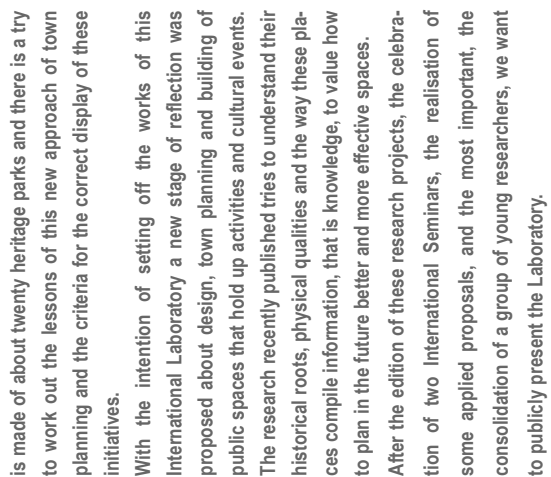

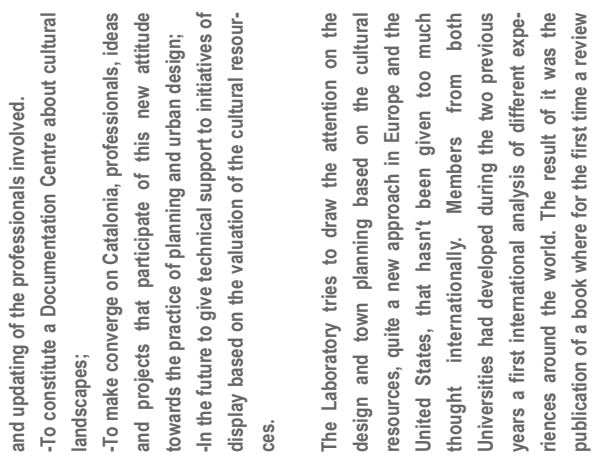

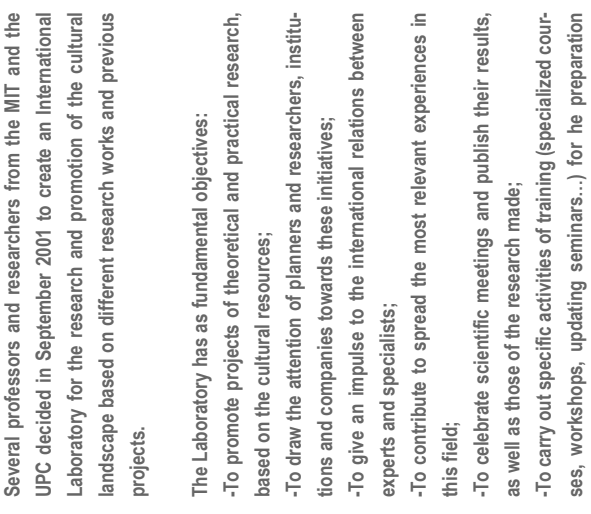


ciudad. Un primer paso sería incrementar la calidad y capacidad de incidencia del diseño y planeamiento urbanístico basado en los recursos culturales. Un objetivo más ambicioso sería el de incentivar, a partir de ahí, el desarrollo económico de antiguos espacios productivos hoy en declive; mejorar la calidad de vida en ciudades y territorios; preservar sus rasgos físicos y culturales distintivos; y alentar modelos de desarrollo más sensibles y efectivos a la hora de combinar la tradición con los requerimientos actuales.

\section{Objetivos}

El Laboratorio tiene como objetivos fundamentales:

- Promover la investigación teórica y aplicada sobre el diseño y planeamiento urbanístico y territorial basado en los recursos culturales, colaborando con las administraciones competentes para adecuar instrumentos y metodologías para conseguirlo.

- Contribuir a la mayor sensibilización para que cualquier intervención sea respetuosa con el paisaje donde se insiere.

- Atraer la atención de proyectistas e investigadores, de instituciones y empresas hacia estas iniciativas. - Impulsar las relaciones internacionales entre expertos y estudiosos y contribuir a divulgar las experiencias de mayor interés en este campo.

- Celebrar encuentros científicos y publicar sus resultados, así como el de las investigaciones realizadas. - Realizar actividades específicas de formación (cursos de doctorado, de maestría, de especialización, talleres, seminarios de actualización...) para preparación y actualización de los profesionales implicados. - Constituir un centro de documentación sobre paisajes culturales.

- Hacer converger en Cataluña profesionales, ideas y proyectos que participen de esta nueva actitud hacia la práctica del planeamiento urbano y territorial.

- Dar apoyo técnico a iniciativas basadas en la valoración de los recursos culturales e impulsar la revaloración de territorios deprimidos donde los recursos culturales puedan convertirse en un factor importante de desarrollo. Igualmente, impulsar la preservación de espacios donde dinámicas urbanizadoras ponen en peligro referentes culturales relevantes.

\section{Organización}

El Laboratorio arrancó desde Barcelona, amparado por el Departamento de Urbanismo y Ordenación

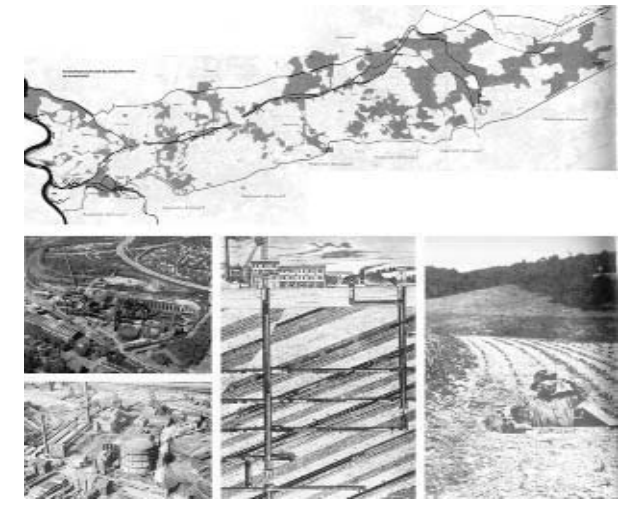


del Territorio y la Universidad Politécnica de Cataluña, en estrecha colaboración con el City Design and Depevelopment Group dentro del Department of Urban Studies and Planning del Massachusetts Institute of Technology, y con el apoyo del Departamento de Universidades, Investigación y Sociedad de la Información del Gobierno de la Generalitat. Desde el inicio se planteó como objetivo fundamental incorporar a otras Universidades, entidades, administraciones y profesionales interesados en participar como miembros o colaboradores de este Laboratorio. Esto ha dado lugar por ejemplo a la consolidación de un proyecto ALFA de la Comisión Europea (Gestión de recursos culturales como fundamento de planes de desarrollo local), en el que participan siete universidades de ambos lados del Atlántico.

No es casual que este Laboratorio Internacional haya sido impulsado en primera instancia por representantes de Cataluña y Massachusetts, porque estos territorios y sus ciudades estan en la vanguardia de los esfuerzos del diseño y planeamiento urbanístico basado en los recursos culturales. Pero al mismo tiempo se pretende integrar en este Laboratorio a diversas instituciones americanas y europeas.

El Laboratorio se planteó con una estructura sencilla de funcionamiento, estableciendo anualmente una agenda de los trabajos a desarrollar por las instituciones universitarias, entidades y administraciones participantes, al objeto de impulsar proyectos y programas de formación.

Pueden formar parte del Laboratorio, a título individual, profesores, estudiosos, proyectistas o gestores, de diversas Universidades e instituciones, en función de su relación con la temática de los paisajes culturales.

Diversas administraciones y entidades ya han dado apoyo (económico e institucional) a los trabajos realizados hasta el momento en el seno del Laboratorio. Otros departamentos universitarios, entidades y administraciones han ofrecido su adhesión o están en fase de integrarse como miembros del Laboratorio.

Entre los Departamentos Universitarios, además del Department of Urban Studies and Planning del Massachusetts Institute of Technology, y del Departamento de Urbanismo y Ordenación del Territorio de la Universidad Politécnica de Cataluña, se han establecido contactos con representantes de las Universidades de Barcelona y Gerona. Se han incorporado asimismo en los trabajos del Laboratorio diversos profesores de las Universidades de Ferrara y Lisboa, en Europa, y de Buenos Aires, Campinas, Córdoba, Montevideo, Recife, Rosario y Santiago de Chile, en Latinoamérica.

Han ofrecido su apoyo a los trabajos del Laboratorio, y en concreto a la realización del Primer Coloquio Internacional "De los sitios memorables a los paisajes culturales; recursos efímeros y construidos al servi-
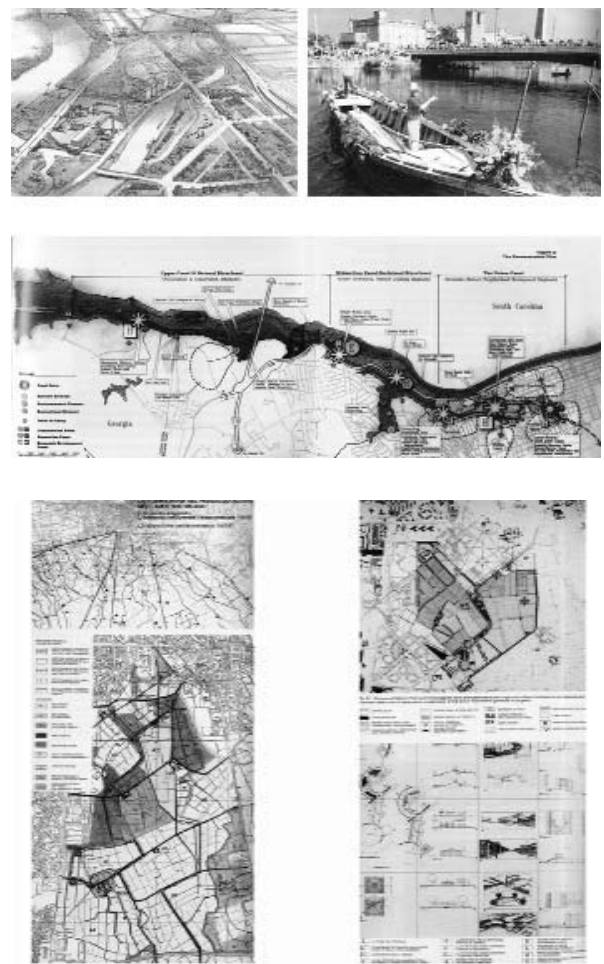
cio del desarrollo local y regional", el Museo Nacional de la Ciencia y la Técnica de Cataluña, el Colegio de Arquitectos de Cataluña y la Agrupación de Arquitectos por la Defensa y Patrimonio Arquitectónico, el Ayuntamiento de Barcelona, el Departamento de Política Territorial de la Generalitat de Cataluña y el Área de Cooperación de la Diputación de Barcelona, así como el City Design and Development Group, la Escuela de Arquitectura y Planeamiento del MIT, y el Programa Office for the Arts Artist-in-Residence.

La dirección y coordinación de los trabajos realizados o impulsados por el Laboratorio corresponden a una Comisión Permanente.

Además se ha constituido un Consejo Asesor para orientar la trayectoria del Laboratorio y ayudar a establecer anualmente la agenda de los trabajos a desarrollar.

Forman parte de la Comisión Permanente del Laboratorio:

- Dennis Frenchman, Professor of the Practice or Urban Design, MIT

- Joaquín Sabaté, Catedrático de Urbanismo de la Universidad Politécnica de Cataluña (coordinador UPC)

- J. Mark Schuster, Professor of Urban Cultural Policy, MIT

- Pere Vall, Profesor Titular de Urbanismo de la Universidad Internacional de Cataluña

Forman parte del Consejo Asesor del Laboratorio diversas personas a título individual o en nombre de las entidades que le dan apoyo, y que tienen en común la relevancia de su tarea en la temática de los paisajes culturales. Estas son hasta el momento las siguientes:

- André Argollo, Profesor Universidad Estadual de Campinas, Brasil

- João Cabral, Profesor de Urbanismo de la Universidade Técnica de Lisboa

- Eusebi Casanelles, Director del Sistema del Museo de la Ciencia y la Técnica de Cataluña

- Eugenio Garcés, Profesor de Urbanismo de la Pontificia Universidad Católica de Chile

- Noemí Goytia, Profesora de Historia de la Universidad Nacional de Córdoba.

- Jordi Ludevid, Presidente Demarcación Barcelona COAC

- Isabel Martínez, Profesora de Urbanismo de la Universidad de Rosario

- Joan Nogué, Catedrático de Geografía Humana de la Universidad de Gerona

- Alicia Novick, Profesora de Historia de la Universidad de Buenos Aires.

- Teresita Nuñez, Profesora de la Universidad de Buenos Aires.

- Antoni Vilanova, Secretario de la Agrupación de Arquitectos por la Defensa del Patrimonio

Forman parte como miembros investigadores del Laboratorio los siguientes profesionales: 
- Pedro Alcocer, arquitecto Universidad de Guadalajara (México) y doctorando UPC

- Álvaro Cuéllar, arquitecto Universidad de Chile y MPU (UPC, Cataluña)

- Jaume Doménech, arquitecto ETSAB (UPC, Cataluña) y doctorando UPC

- Annalisa Giocoli, arquitecta Universidad de Nápoles (Italia) y MPU (UPC, Cataluña)

- Carlos Teodoro Itriago, arquitecto Universidad Central de Venezuela (Venezuela) y doctorando UPC

- Miquel Martí, ingeniero de caminos, canales y puertos, MPU y doctor en Urbanismo por la UPC

- Jorge Mora, arquitecto Pontificia Universidad Católica de Chile y doctorando UPC

- Ana Carolina Pagliuso, arquitecta paisajista, Curitiba (Brasil) y doctoranda UPC

- Leonel Pérez, arquitecto, Profesor de la Universidad de Concepción (Chile) y doctorando UPC

- Doris Tarchopulos, arquitecta, directora de investigación de la Universidad Javeriana de Bogotá (Colombia) y doctoranda UPC

- Anna Trillo, arquitecta Universidad de Nápoles (Italia)

\section{Primeros resultados}

A lo largo de algo más de tres años de vida el Laboratorio ha impulsado diversas iniciativas. Entre éstas cabe destacar la presentación de ponencias en diversos Seminarios y la publicación por parte de sus miembros de numerosos artículos sobre la temática de los paisajes culturales, así como de tres libros colectivos: Designing the Llobregat Corridor. Cultural Landscape and Regional Development. Projectant l'eix del Llobregat. Paisatge cultural i desenvolupament regional. Universidad Politécnica de Cataluña y Massachusetts Institute of Technology. Barcelona, 2001.

Patrimonio y desarrollo territorial. Colonias, Sèquia de Manresa y Delta del Llobregat. Diputación de Barcelona. Universidad Politécnica de Cataluña. Barcelona, 2004.

Llocs amb Esdeveniments. Event Places. Universidad Politécnica de Cataluña y Massachusetts Institute of Technology. Barcelona, 2004.

Entre los trabajos profesionales que han realizado los integrantes del Laboratorio por encargo fundamentalmente de la Diputación de Barcelona, estarian la ordenación del corredor fluvial del río Cardener; la valoración de los recursos patrimoniales, de la estructura y ordenación de los ríos Llobregat, Anoia y Cardener, y el plan Especial del Parque Agrario del Baix Llobregat. 
Un proyecto de investigación conjunto de dos equipos del MIT y de la UPC durante dos años tuvo su colofón en enero de 2004 con un Coloquio Internacional "De los sitios memorables a los paisajes culturales. Recursos efimeros y construidos al servicio del desarrollo local y regional". Dicho Coloquio se planteó el objetivo de presentar el resultado del trabajo de diversos estudiosos y especialistas, tanto nacionales como internacionales, así como de los protagonistas de proyectos de impulso a escala urbana y territorial basados en los recursos patrimoniales.

Poco antes había tenido lugar en el Massachusetts Institute of Technology la celebración de un Foro sobre sitios memorables con la participación durante varios meses de protagonistas de diferentes eventos relevantes en aquel país.

Desde inicios de 2004 cuatro Universidades latinoamericanas (Nacional de Córdoba, de Buenos Aires, de la República de Montevideo y Pontificia Católica de Santiago de Chile) y tres europeas (Ferrara, Lisboa y Politécnica de Cataluña) desarrollan en continuidad con los trabajos del Laboratorio un proyecto ALFA financiado por la Comunidad Europea. Dicho proyecto, denominado Gestión de recursos culturales como fundamento de planes de desarrollo de base local, tiene entre otros los siguientes objetivos:

- Integrar una red de investigación internacional sobre dicha temática

- Impulsar la elaboración conjunta de Programas de Doctorado y de Maestría

- Proponer políticas e instrumentos de gestión de recursos culturales para el desarrollo local

- Impulsar experiencias piloto de desarrollo local basada en los recursos patrimoniales

- Gestionar ante las instituciones correspondientes la implementación de estudios específicos sobre la gestión de los recursos culturales.

En paralelo se han desarrollado diversas actividades docentes (cursos de grado y de doctorado) y ha arrancado un primer Programa de Postgrado sobre Paisajes Culturales, Patrimonio y Proyecto territorial.

Finalmente, y esto es lo más relevante, ya que asegura la continuidad de la trayectoria del Laboratorio, varios profesionales, que cursan su Programa de doctorado (en la UPC y el MIT) están desarrollando sus investigaciones, algunas bien avanzadas, en este campo.

Es a partir de estas primeras iniciativas, que nos atrevemos a dar a conocer públicamente este Laboratorio. 